\title{
Lower Plasma Levels of IL-35 in Patients with Primary Biliary Cirrhosis
}

\author{
Tengda Li, ${ }^{1, *}$ Yuanlan Huang, ${ }^{2, *}$ Peng Liu, ${ }^{1}$ Yun Liu, ${ }^{3}$ Jie Guo, ${ }^{3}$ Weiwei Zhang, ${ }^{3}$ \\ Mingli Gu, ${ }^{3}$ Cheng Qian ${ }^{4}$ and Anmei Deng ${ }^{1}$

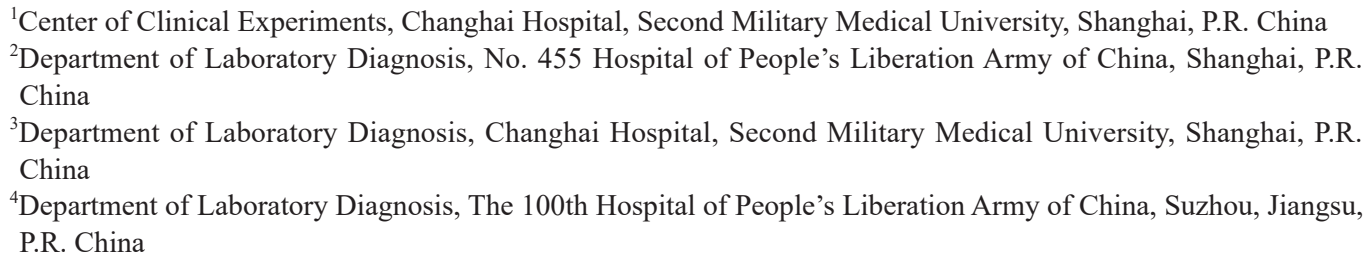

Primary biliary cirrhosis (PBC) is an autoimmune liver disease. Its histological characteristics, such as progressive intrahepatic bile duct destruction, cholestasis, and liver cirrhosis, are caused by the body's autoimmune disorders. Interleukin (IL)-35 has two subunits (p35 and Ebi3) and is a member of the IL-12 family of heterodimeric cytokines. IL-35 has immunosuppressive functions and plays an important role in many autoimmune diseases. In this study, we compared plasma levels of IL-35 and relative mRNA expression levels of p35 and Ebi3 in peripheral blood mononuclear cells (PBMCs) from 70 PBC patients and 70 healthy individuals. The results showed that the relative expression levels of Ebi3 mRNA were lower in PBMCs from PBC patients than in PBMCs from healthy individuals, whereas the levels of p35 mRNA were similar in both groups. Plasma IL-35 concentrations were lower in patients with PBC than in healthy individuals. Plasma levels were higher in PBC patients at an advanced stage compared to patients at an early stage. Variable plasma levels with different stages were also found in transforming growth factor beta (TGF- $\beta$ ), which is mainly produced by regulatory T cells (Tregs). IL-35 and TGF- $\beta$ levels were positively correlated with each other, and IL-35 was capable of promoting the inhibitory functions of Tregs in PBC patients at both the early and late stages of disease. Lower plasma IL-35 levels were accompanied by higher levels of typical clinical parameters, such as alkaline phosphatase, or of proinflammatory cytokines, such as interferon-gamma (IFN- $\gamma$ ), in PBC patients ( $P<0.05$ for each). We propose that IL-35 may be involved in the pathogenesis of PBC and could be a potential biomarker for diagnosing this disease.

Keywords: IFN- $\gamma$; IL-23; IL-35; primary biliary cirrhosis; TGF- $\beta$

Tohoku J. Exp. Med., 2018 February, 244 (2), 123-131. (C) 2018 Tohoku University Medical Press

\section{Introduction}

Primary biliary cirrhosis (PBC) is a typical autoimmune liver disease. This fibrotic disease is characterized histologically by progressive intrahepatic bile duct destruction and serologically by high titers of autoantibodies against the E2 component of the pyruvate dehydrogenase enzyme (Webb et al. 2015). The E2 component is found on the inner mitochondrial membrane; therefore, autoantibodies against the E2 component are called anti-mitochondrial antibodies (AMAs) (Webb et al. 2015). Women over 40 years of age are the most susceptible population for PBC, with an incidence of $1 / 1,000$ specifically (Boonstra et al. 2012). Prevalence of $P B C$ varies between regions, with a rate of 49 per 100,000 adults in China (Griffiths et al. 2014). Currently, the only approved treatment for PBC is ursodeoxycholic acid (UDCA), which can delay histological progression and improve prognosis. However, around $25 \%$ of patients are unresponsive to this drug (Imam and Lindor 2014; Carey et al. 2015). Treatment failure can result in

Received August 17, 2017; revised and accepted January 10, 2018. Published online February 15, 2018; doi: 10.1620/tjem.244.123.

*These two authors contributed equally to this work.

Correspondence: Anmei Deng, Ph.D., Center of Clinical Experiments, Changhai Hospital, Second Military Medical University, \#168

Changhai Road, Yangpu Dist., Shanghai 200433, P.R. China.

e-mail: amdeng70@163.com

Cheng Qian, Ph.D., Department of Laboratory Diagnosis, The 100th Hospital of People's Liberation Army of China, \#4 Canglang

Pavilion St., Suzhou 215007, P.R. China.

e-mail: qiancheng824@163.com 
bile duct injury and liver cirrhosis, necessitating liver transplant (Corpechot et al. 2008).

CD4+ T cells, including T helper type 1 (Th1) cells, type 17 (Th17) cells, and regulatory T cells (Tregs), are the main immune cells involved in the immunopathology of PBC (Fenoglio et al. 2012; Webb et al. 2015). Th1 cells mainly produce cytokines such as interferon gamma (IFN$\gamma$ ), whereas Th17 cells mainly secrete IL-23, IL-17, IL-6, and others. Tregs help regulate the body's immune system and prominently secrete immune molecules such as transforming growth factor beta (TGF- $\beta$ ) (Fenoglio et al. 2012; Raphael et al. 2015).

Several pieces of evidence demonstrate the functions of CD4+ T cells in the pathogenesis of PBC. For example, biliary duct lesions in $\mathrm{PBC}$ patients showed elevated mRNA expression levels of IFN- $\gamma$ (Shindo et al. 1996). Fibrosisassociated cytokines, such as TGF- $\beta$ (Rosenbloom et al. 2010 ), contributed to the fibrotic process of PBC and affected the formation and function of Tregs and effector $\mathrm{T}$ lymphocytes (Th17 cells), which mainly produce interleukin (IL)-23, IL-6, and IL-17 (Fenoglio et al. 2012). Tregs, which secrete TGF- $\beta$, play an important role in preventing autoimmune reactions (Filaci et al. 2011; Yamaguchi et al. 2011; Sakaguchi et al. 2012). PBC is characterized by a Treg-Th17 cell imbalance, as evidenced by the increased IL-23 and IL- 6 but decreased TGF- $\beta$ expression levels in plasma from patients with PBC (Rong et al. 2009). Th1 cells are important in this disease and can form an IL-12 expression feedback loop through IFN- $\gamma$ (Webb et al. 2015).

IL-35 is a member of the IL-12 family of heterodimeric cytokines, which also includes IL-12, IL-23, and IL-27 (Guan et al. 2017). All four cytokines are composed of two subunits. IL-35 consists of p35 and Ebi3, and IL-12 is composed of p35 and p40 (Guan et al. 2017). IL-35 has immunosuppressive functions (Vignali and Kuchroo 2012). Ectopic expression of IL-35 conferred IL-35 regulatory activity on naïve $T$ cells, whereas recombinant IL-35 inhibited T-cell proliferation (Collison et al. 2007). Thus, IL-35, which was probably released by Tregs, was critical for maximal immunosuppressive activity in the body (Collison et al. 2007). Recently, some reports showed that exposure to IL-35 induced the proliferation of CD4+CD25+Foxp3 T cells, which led to the increased expression of IL-10 and inhibition of functional T cells (Niedbala et al. 2007). Th17 cells are known to be critical in the pathology of PBC, but their function can also be disrupted by exposure to recombinant IL-35. For example, T cells from Ebi0 $0^{-/}$mice produced greater amounts of IL-17 than were produced by T cells from wild-type mice (Niedbala et al. 2007; Wirtz et al. 2011).

Because IL-12 family members, including IL-35, are composed of more than one subunit, their receptors on immune cells also involve multiple subunits (Collison et al. 2012; Vignali and Kuchroo 2012). Collison et al. demonstrated that the expression of gp130 and IL-12 receptor subunit beta 2 (IL12R $\beta 2$ ) on T cells enabled transduction of the
IL-35 signal, indicating the role of these molecules as receptors for IL-35 (Collison et al. 2012). However, it remains to be investigated whether IL-35 receptors are expressed in hepatocytes or cholangiocytes at the cellular level.

Despite increasing research into the pathogenesis of PBC, the function of IL-35 in PBC is not fully understood. Hence, we tested the protein level of IL-35 and relative mRNA levels of p35 and Ebi3 in blood samples from patients with $\mathrm{PBC}$ or healthy individuals. We analyzed the correlation of IL-35 levels with the levels of important cytokines involved in PBC, such as IFN- $\gamma$ and IL-17, to reveal the roles of IL-35 in the pathogenic process of this disease.

\section{Methods}

Subjects

In this retrospective study, peripheral blood samples were obtained from patients with PBC and from healthy individuals at Changhai Hospital and ChangZheng Hospital affiliated with the Second Military Medical University, Shanghai, China between September, 2011 and February, 2017. This study included 70 patients with PBC (55 women, 15 men; mean age: 45.59 years, range: 22-60 years), designated as the $\mathrm{PBC}$ group. Standards for $\mathrm{PBC}$ diagnosis were as follows: 1) high serum levels of alkaline phosphatase (ALP) and gamma-glutamyl transferase (GGT), 2) hepatic histological reports consistent with $\mathrm{PBC}$, and 3) positive testing for AMAs. Patients were excluded if they had hepatitis of other known causes, such as viral infection or alcoholism.

Histological features of different stages of PBC were as follows. Stage I featured interlobular cholangitis in the portal areas. Stage II featured disappearance of interlobular bile ducts, proliferation of small bile ducts, and infiltration of inflammatory cells around portal areas. Stages III and IV featured disappearance of the typically damaged bile ducts and presence of regenerative nodules, liver cirrhosis, or progressive fibrosis. Histological changes of the adjacent stages might also be found in stages III and IV. Patients were equally distributed in terms of histological stage of disease (17 patients in stage I, 15 patients in stage II, 18 patients in stage III, and 20 patients in stage IV). We ascertained whether patients were being treated with UDCA when we collected their blood samples. There were 36 patients without UDCA and 34 patients with UDCA treatment in this study.

The healthy control group comprised 70 healthy individuals (53 women, 17 men; mean age: 45.13 years, range: $24-60$ years). These individuals did not have any hepatic or other disease that could influence the examined results. Age and sex distributions of the control group were similar to those of the PBC group $(P>0.05)$. This study was approved by the medical research ethics committee of the Second Military Medical University. Written informed consent was obtained from all subjects prior to enrollment.

\section{Separation of PBMCs and plasma}

Blood samples were centrifuged at $1,800 \times g$ for $10 \mathrm{~min}$ to obtain plasma. For PBMCs, the original blood was diluted by phosphate-buffered saline ( $\mathrm{pH}$ 7.2-7.4, ThermoFisher Scientific, USA) in two folds and added to a tube containing Ficoll-Hypaque solution (Sigma, USA), which was centrifuged at $400 \times g$ for 30 minutes to 
obtain PBMCs. Finally, PBMCs were washed in PBS twice and resuspended to the proper concentration for further analysis.

\section{Total RNA isolation and $q R T-P C R$}

PBMCs were mixed with chloroform and centrifuged at 12,000 $\times g$ for 5 min at $4{ }^{\circ} \mathrm{C}$. The water phase was separated out, mixed with isopropyl alcohol, and set at room temperature for $30 \mathrm{~min}$. Sediments were collected after centrifuging the mixture at $12,000 \times \mathrm{g}$ for $15 \mathrm{~min}$ at $4^{\circ} \mathrm{C}$. Precooled $75 \%$ alcohol was added to the precipitates and centrifuged at $6,000 \times \mathrm{g}$ for $5 \mathrm{~min}$ at $4^{\circ} \mathrm{C}$. After drying at room temperature for 2 to $5 \mathrm{~min}$, the RNAs were resuspended in RNase-free water. Concentration and purification were tested on a Nanodrop 2000 (Thermo, USA). Ideal samples for qRT-PCR had an absorbance ratio at $260 \mathrm{~nm} / 280 \mathrm{~nm}$ exceeding 1.8 .

Subsequently, the One-Step RT-PCR kit (Takara, China) was used to examine the relative mRNA levels of targeted genes. Experimental steps were carried out according to the manufacturer's protocol. Primers used in these experiments are as follows. Reference mRNA was $\beta$-actin (F: 5'-GTGGCCGAGG ACTTTG ATTG-3', R: 5'-CCTGTAACAA CGCATCTCAT ATT-3'). Targeted mRNAs were IL12A (p35) (F: 5'-CCTTCACCAC TCCCAAAAC-3', R: 5'-TGTCTGGCCT TCTGGAGCAT-3') and Ebi3 (F: 5'-TCCTT CATTG CCACGTACAG-3'，R: 5'-GCTCTGTTAT GAAAGG CACG-3'). Expression levels of targeted mRNAs were measured by the relative qualification method.

\section{ELISA for cytokines in plasma}

Protein expression levels of IL-35 were measured by a human IL-35 ELISA kit (ARG81146, Arigo Co., Shanghai, China). Levels of other cytokines in plasma samples from patients with $\mathrm{PBC}$ and healthy individuals were also measured by ELISA kits (Arigo Co.). All procedures were performed in accordance with the manufacturers' protocols.

\section{Statistical analysis}

Results are reported in the form of median [range] or mean \pm standard deviation. Measured data from the PBC and healthy control groups were compared by using $t$-tests with two independent samples or nonparametric Mann-Whitney tests. The correlation of two variables in these experiments was calculated by Pearson correlation analysis. All statistical analysis was performed by using IBM SPSS Statistics 21.0. The test level was 0.05 .

\section{Results}

Plasma level of IL-35 and relative $m R N A$ level of $p 35$ or Ebi3 in PBMCs

The IL-35 protein levels in plasma samples were 2.95 [0.24-5.63] $\mathrm{ng} / \mathrm{ml}$ for patients with $\mathrm{PBC}$ and 8.21 [0.89$17.03] \mathrm{ng} / \mathrm{ml}$ for healthy individuals (Mann-Whitney test $Z$ $=-9.027, P<0.0001$ ) (Fig. 1a). Patients with PBC were further divided into subgroups according to their histological stage of disease (Fig. 1b). Results of the independentsamples Kruskal-Wallis test showed that IL-35 concentration in plasma from patients with stage III or IV disease was greater than that in plasma from patients with stage II disease $(H=33.916, P<0.0001$ for both; $P=0.003$ and $<0.0001$ separately). Plasma IL-35 concentration was greater for UDCA-treated patients than for patients without this treatment $(Z=-4.830, P \leq 0.0001$; Fig. $1 \mathrm{c})$.

Results of qRT-PCR analysis revealed that relative mRNA levels of Ebi3 in PBMCs were 0.46 [0.05-1.03] for patients with $\mathrm{PBC}$ and 1.17 [0.52-1.76] for healthy individuals $(Z=-9.556, P<0.0001)$ (Fig. $1 \mathrm{~d})$. The mRNA levels of p35 were 1.13 [0.08-1.80] for patients with $\mathrm{PBC}$ and 1.14 [0.55-1.68] for healthy controls, with no statistical difference between the groups $(Z=-0.846, P=0.398$; Fig. 1e).

\section{Correlation of plasma IL-35 expression with clinical parameters}

Table 1 reports plasma concentrations of clinical parameters for patients with PBC and healthy individuals. Analyses of correlations between plasma IL-35 concentration and clinical variables in patients with PBC (Fig. 2a-d) revealed negative correlations of IL-35 with ALP $(r=$ $-0.467, P<0.0001)$, GGT $(r=-0.345, P=0.003)$, AST $(r$ $=-0.391, P=0.001)$, and ALT $(r=-0.563, P<0.0001)$, but no correlation with the concentration of total bilirubin $(r=$ $-0.032, P=0.794), \operatorname{IgG}(r=-0.129, P=0.288)$, or $\operatorname{IgM}(r$ $=-0.038, P=0.754)$.

\section{Correlation of IL-35 with cytokines in plasma}

ELISA results of cytokine protein levels in plasma samples from patients with $\mathrm{PBC}$ vs. healthy individuals were as follows: IL-23: 185.68 [94.92-279.34] $\mathrm{pg} / \mathrm{ml}$ vs. 72.51 [30.97-124.95] pg/ml $(Z=-10.106, P<0.0001)$ (Fig. 3c), IL-17: 26.83 [7.11-47.50] pg/ml vs. 8.05 [5.10-13.96] $\mathrm{pg} / \mathrm{ml}(Z=-9.819, P<0.0001)$ (Fig. 3e), and IL-6: 476.63 [200.94-672.06] pg/ml vs. 163.57 [10.17-296.77] pg/ml $(Z$ $=-9.948, P<0.0001)$. The IFN- $\gamma$ concentration in PBC patients vs. healthy individuals was $220.33 \pm 43.37 \mathrm{pg} / \mathrm{ml}$ vs. $71.33 \pm 29.41 \mathrm{pg} / \mathrm{ml}(t=23.789, P<0.0001)$ (Fig. 3a). The IL-10 level in patients with PBC vs. healthy individuals was 700.47 [305.21-1190.57] pg/ml vs. 56.26 [28.08$100.18] \mathrm{pg} / \mathrm{ml}(Z=-10.211, P<0.0001)$.

To analyze the TGF- $\beta$ concentration, which plays different roles in different stages of PBC, we subdivided patients into early (stages I-II, $\mathrm{n}=32$ ) and late (stages III-IV, $\mathrm{n}=38$ ) stages of disease. Independent-samples Kruskal-Wallis test for TGF- $\beta$ concentration showed differences between the healthy control, early-stage, and latestage groups $(H=76.371, P<0.0001)$. The TGF- $\beta$ concentration in the control group was higher than in the earlystage group $(P=0.004)$ but lower than in the late-stage group $(P<0.0001)$. The concentration in the late-stage group was higher than in the early-stage group $(P<0.0001)$ (Fig. 3g).

Pearson correlation analysis showed that the protein level of IL-35 in plasma samples from patients with PBC was negatively correlated with levels of IFN- $\gamma(r=-0.557$, $P<0.0001$; Fig. 3b), IL-23 ( $r=-0.318, P=0.007$; Fig. 3d), and IL-17 ( $r=-0.381, P=0.001$; Fig. $3 f)$, and positively correlated with TGF- $\beta$ concentration in the early $(r=0.499$, $P=0.004)$ and late $(r=0.512, P=0.001)$ stages of disease (Fig. 3h, i). Plasma IL-35 concentration showed no signifi- 


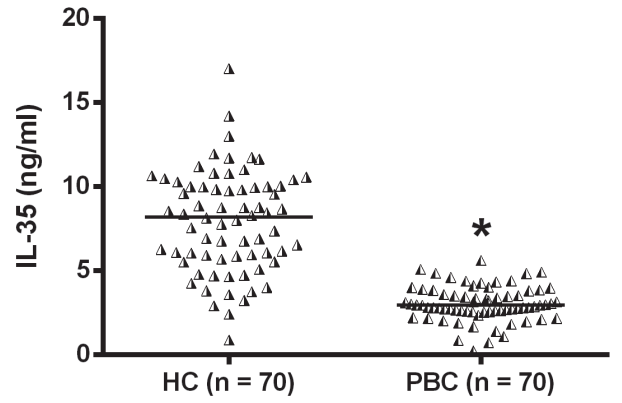

$\mathrm{c}$

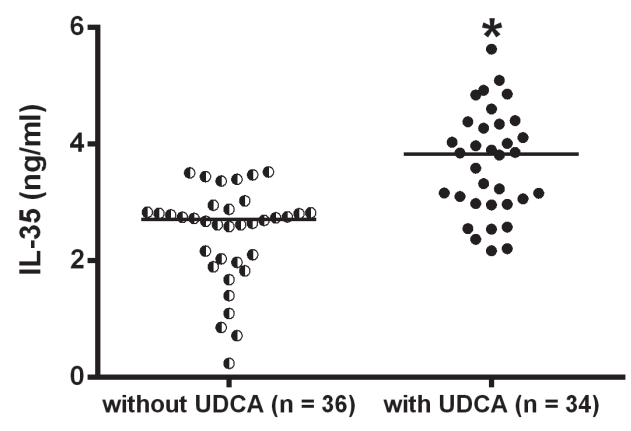

e

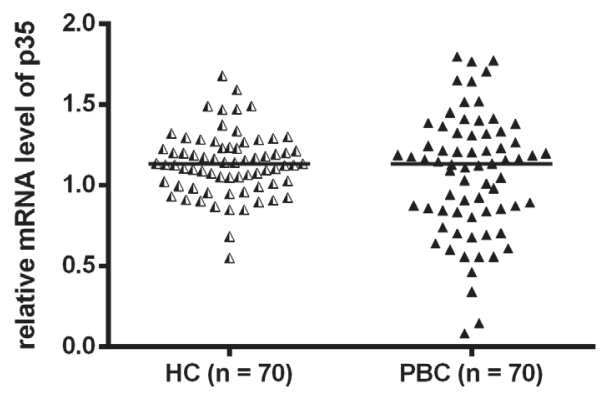

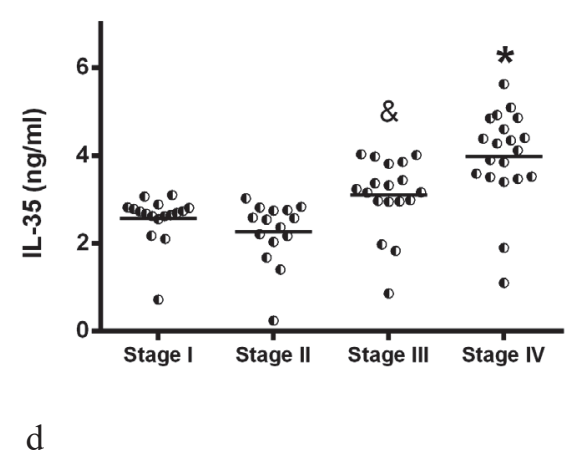

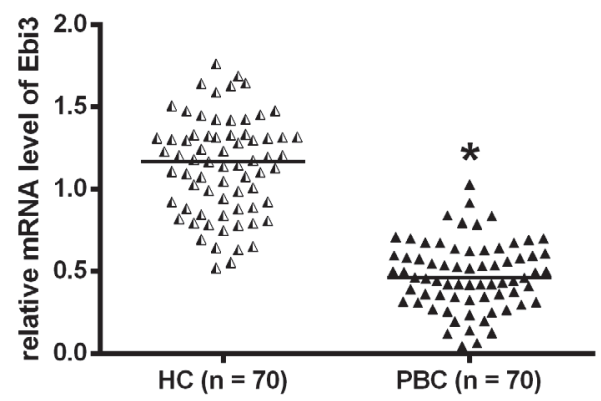

ig. 1. Plasma level of IL-35 and relative mRNA expression level of p35 or Ebi3.

Plasma levels of IL-35 in: (a) PBC patients and HC, $* P<0.0001$ vs. HC; (b) patients in different stages, $* P<0.0001$ vs. stage II, ${ }^{\&} P<0.01$ vs. stage II; (c) patients with or without UDCA treatment, $* P<0.0001$ vs. without UDCA. Relative mRNA levels of: (d) Ebi3 and (e) p35 in PBMCs from PBC and HC, ${ }^{*} P<0.0001$ vs. HC. PBC, primary biliary cirrhosis; HC, healthy controls.

Table 1. Clinical parameters for patients with primary biliary cirrhosis (PBC) and healthy controls (HC).

\begin{tabular}{llllllll}
\hline Group & ALP (U/L) & AST (U/L) & ALT (U/L) & GGT (U/L) & TBIL $(\mu \mathrm{mol} / \mathbf{L})$ & IgG (g/L) & IgM (g/L) \\
\hline PBC (n=70) & $414.83 \pm 156.39^{*}$ & $116.99 \pm 56.67^{*}$ & $90.96 \pm 48.04^{*}$ & $445.96 \pm 198.32^{*}$ & $52.91 \pm 9.03^{*}$ & $20.42 \pm 2.15^{*}$ & $3.08 \pm 0.45^{*}$ \\
HC (n=70) & $76.99 \pm 35.22$ & $25.11 \pm 8.60$ & $20.23 \pm 8.44$ & $37.89 \pm 15.15$ & $14.77 \pm 6.20$ & $13.14 \pm 1.72$ & $2.42 \pm 0.46$ \\
\hline
\end{tabular}

Data are reported as the mean $\pm \mathrm{SD}$. $* P<0.0001$ vs HC. ALP, alkaline phosphatase; AST, aspartate aminotransferase; ALT, alanine aminotransferase; GGT, gamma glutamyltransferase; TBIL, total bilirubin; Ig, immunoglobulin.

cant relationship with the concentration of IL-6 $(r=0.102$, $P=0.402)$ or IL10 $(r=-0.021, P=0.862)$.

\section{Discussion}

$\mathrm{PBC}$ is an idiopathic cholangitis accompanied by pro- 
a

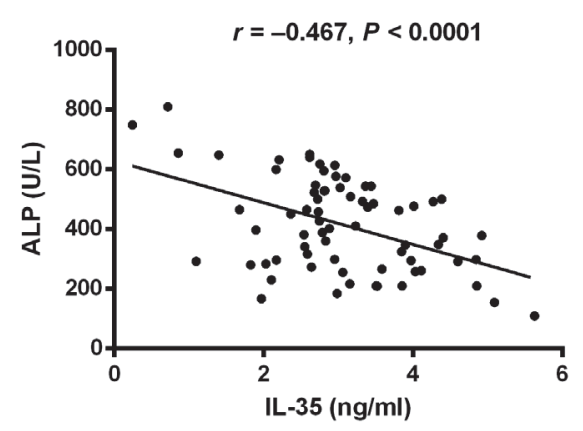

c

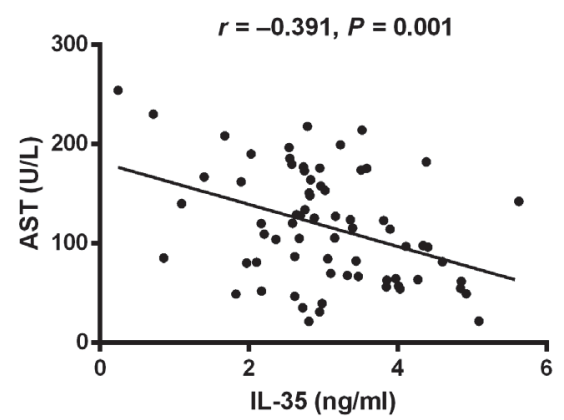

b

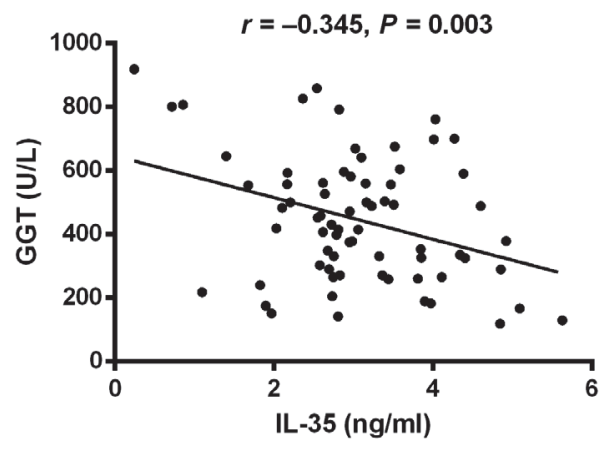

d

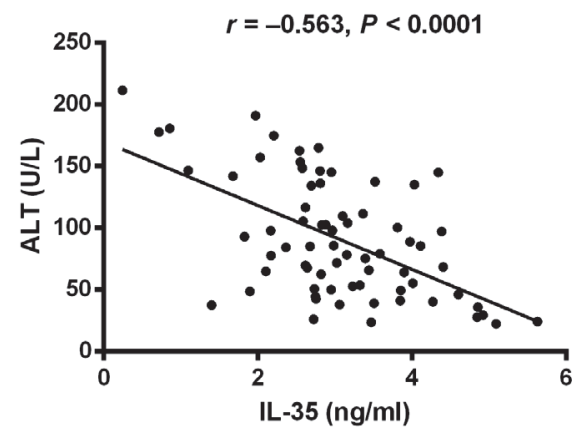

Fig. 2. Correlation of plasma IL-35 concentration with key clinical parameters.

Correlation of plasma IL-35 concentration with (a) ALP, (b) GGT, (c) AST, and (d) ALT levels in patients with primary biliary cirrhosis.

gressive intrahepatic bile duct destruction, cholestasis, and liver cirrhosis (Lleo et al. 2014). We found that the IL-35 plasma concentration was lower for patients with $\mathrm{PBC}$ than for healthy controls. Relative mRNA expression of Ebi3 was lower in patients than in controls, which implies that the Ebi3 subunit might be involved in PBC development. We did not observe a difference in relative mRNA expression of $\mathrm{p} 35$ between the two groups, as was also the case in our previous research (Qian et al. 2013). This result might be due to the complexity of IL-12 cytokines. Specifically, IL-35 belongs to the IL-12 family that contains IL-23, IL-27, and IL-12 (Guan et al. 2017). All four of these molecules consist of an $\alpha$ chain (p19, p28, or p35) and a $\beta$ chain (p40 or Ebi3) (Pylayeva-Gupta 2016; Guan et al. 2017), and some of them share the same subunits. For example, IL-12 shares p35 with IL-35; thus, the expression of p35 is influenced by both IL-35 and IL-12 (Pylayeva-Gupta 2016; Guan et al. 2017).

Early in our work, we found that the plasma levels of IL-12 did not differ between PBC patients and healthy individuals. Considering that IL-12 contains the subunit $\mathrm{p} 35$, the results presented in this article are probably reasonable. Plasma IL-35 concentration varied depending on the disease stage and use of UDCA treatment, which suggests that IL-35 expression might be related to the severity of this dis- ease. Finally, the plasma IL-35 concentration was negatively related to concentrations of clinical parameters of disease activity (ALP, GGT, AST, ALT) and cytokines produced by CD4+ T cells (IFN- $\gamma$, IL-23, IL-17), and was positively related to the Treg-related cytokine TGF- $\beta$ in patients at different stages of disease.

As important immune cells, CD4+ T cells are involved in the pathogenesis of PBC (Hirschfield and Gershwin 2013; Wang et al. 2015a; Webb et al. 2015). One paper reported that the concentration of Th1-related IFN- $\gamma$ was increased in PBC patients but decreased after UDCA treatment, indicating the relationship of this cytokine with disease activity (Saeki et al. 1995). PBC also involves expression of TGF- $\beta$, which regulates the differentiation of Th17 cells and Tregs (Rosenbloom et al. 2010; Fenoglio et al. 2012; Liu et al. 2013; Waluga et al. 2015). The Th17-Treg balance is impaired in PBC (Fenoglio et al. 2012). Th17 cells mainly secrete IL-17, IL-6, and IL-23 and have been associated with the pathologies of several autoimmune diseases, including multiple sclerosis, rheumatoid arthritis (RA), and inflammatory bowel disease (Miao et al. 2014; Alunno et al. 2015; Ueno et al. 2015; Dos Passos et al. 2016). Patients with PBC have greater numbers of IL-17+ cells than healthy controls, and immune dysregulation in $\mathrm{PBC}$ is related to preferential activation of Th17 cells (Lan 
a

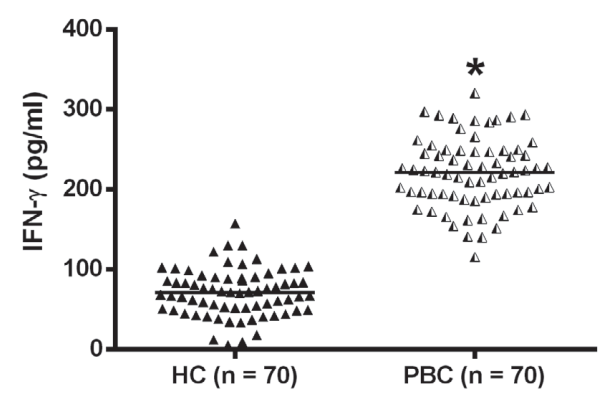

c

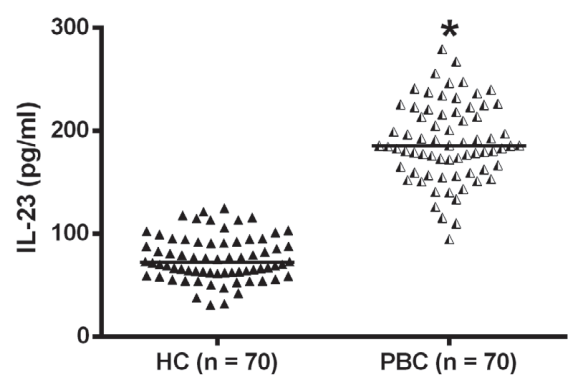

e
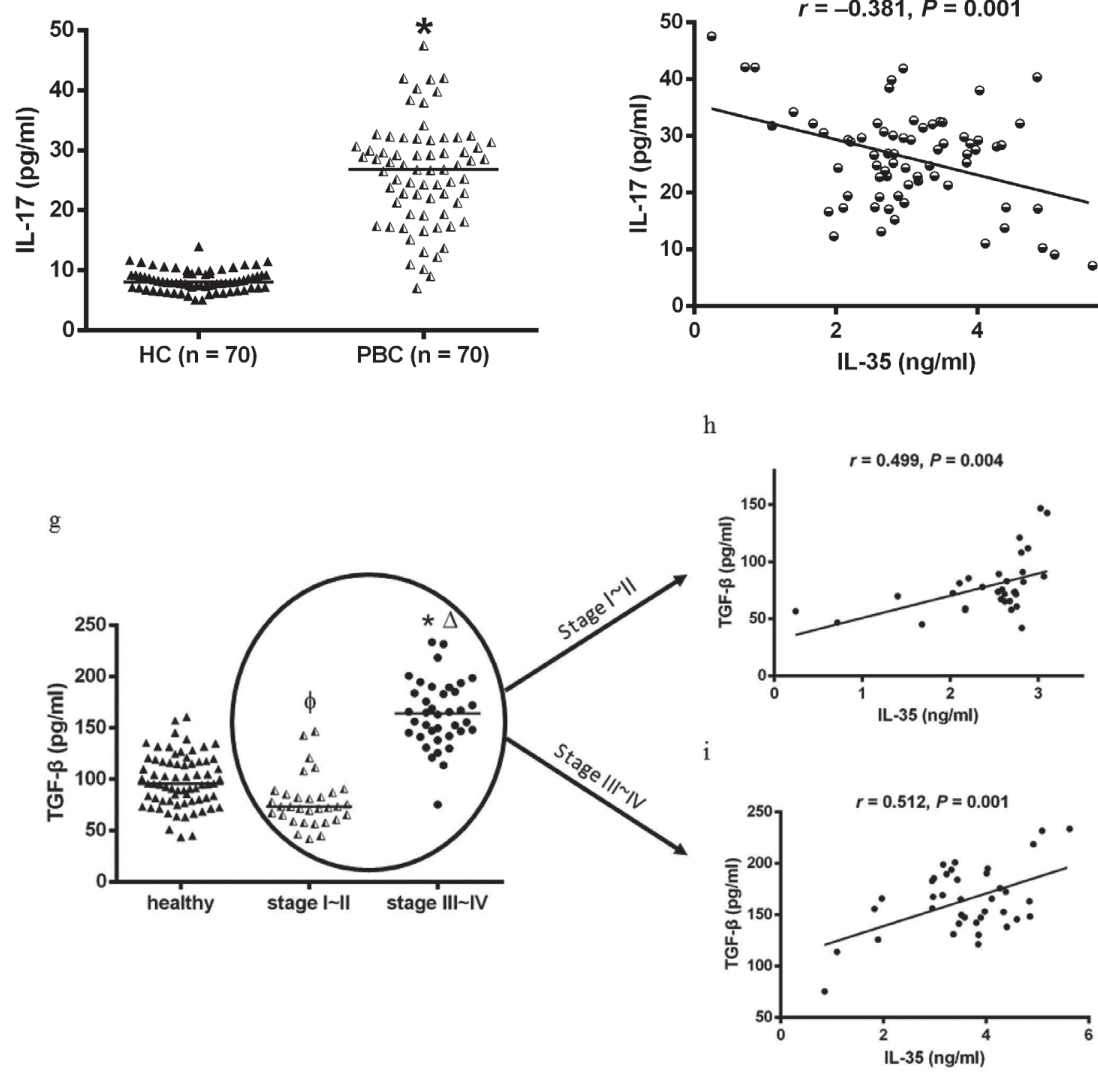

b

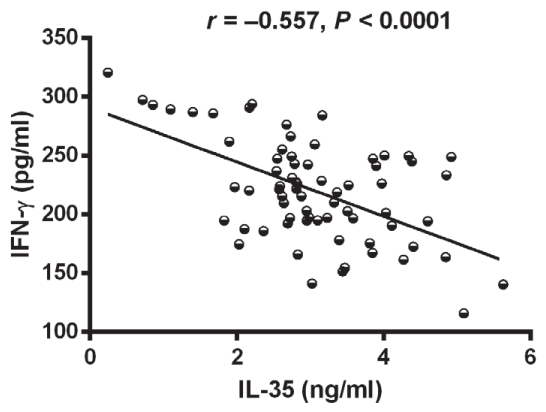

d

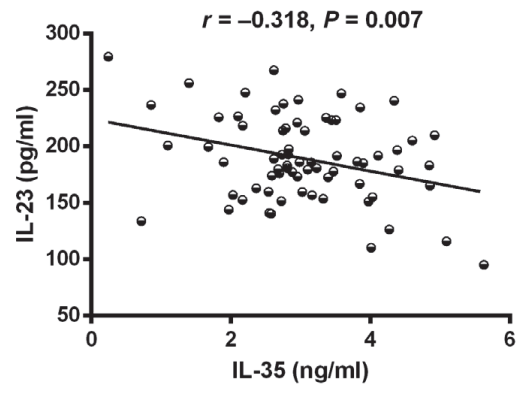

f

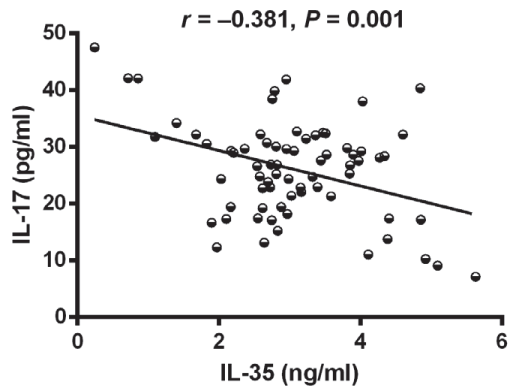


et al. 2009). Previous reports emphasized the role of Tregs in maintaining self-tolerance and preventing autoimmune disease (Kleinewietfeld and Hafler 2013). Patients with PBC showed below-normal numbers of circulating CD4+CD25 $5^{\text {high }}$ Tregs (Wang et al. 2010; Wang et al. 2015b), and UDCA treatment for 1 year increased these numbers (Lacerda et al. 1995). Rong et al. (2009) reported increased IL-23 and IL- 6 and decreased TGF- $\beta$ concentrations in the circulation of patients with PBC. Taken together, these findings highlight the activation of Th17 and inhibition of Tregs in PBC (Rong et al. 2009). This explanation is supported by evidence that $\mathrm{PBC}$ patients show decreased numbers of Foxp3+ Tregs in peripheral blood, and that IL-17+ cells accumulated around damaged bile ducts (Fenoglio et al. 2012).

Expression levels of IFN- $\gamma$, IL-23, IL-17, and IL-6 in plasma were higher in patients with $\mathrm{PBC}$ than in healthy individuals. This result was consistent with previous research. The plasma TGF- $\beta$ concentration in patients with early-stage $\mathrm{PBC}$ was lower than the concentration in healthy individuals, consistent with the Th17-Treg imbalance in PBC. However, the concentration was higher in patients with late-stage $\mathrm{PBC}$ than in healthy individuals, a result that seems to be different from those of Rong et al. (2009). Previous studies showed that TGF- $\beta$ and IL-6 expression levels are elevated in the fibrotic process in $\mathrm{PBC}$, whereas the inflammatory process involves decreased TGF- $\beta$ and increased Th17 expression levels (Fenoglio et al. 2012). These previous results might help explain the different expression levels of TGF- $\beta$ in the early and late stages of disease, as the early stage is mainly characterized by inflammation whereas the late stage is characterized by progressive liver cirrhosis (Fenoglio et al. 2012).

Interestingly, we found that the protein level of IL-35 in plasma had the same tendency as TGF- $\beta$. IL-35 and TGF- $\beta$ are mainly produced by Tregs and could promote the inhibitory functions of Tregs for maintaining the immune balance in the body (Fenoglio et al. 2012; Kleinewietfeld and Hafler 2013; Pylayeva-Gupta 2016). To some degree, these cytokines have similar functions and probably are synergistic. This relationship is supported by their positive correlations with each other in the early and late stages of disease, as shown in Fig. 3h-i. Finally, the elevated plasma concentration of IL-10 from PBC patients might indicate that this cytokine was not only influenced by Tregs, but also by Th2, Th17, and other innate immune cells, such as macrophages (Moore et al. 2001; Mannino et al. 2015; Kumar et al. 2016).

IL-35 is an immunosuppressive cytokine that inhibits T-cell activity in a cell contact-dependent manner (Pylayeva-Gupta 2016). IL-35 is not detected in naïve T cells, but inflammatory signals induce its expression by differentiated T cells, such as Tregs (Collison et al. 2010; Li et al. 2012). Tregs from $I l 12 a^{-/-}$or $\mathrm{Ebi3}^{-/-}$mice showed reduced capacity to inhibit $\mathrm{T}$ cell proliferation (Mattner et al. 1996; Nieuwenhuis et al. 2002). Exposure to IL-35 induced the proliferation of CD4+CD25+Foxp $3+\mathrm{T}$ cells, the expression of IL-10, and the functional inhibition of effector T cells. Using a collagen-induced arthritis model, researchers showed that recombinant IL-35 induced proliferation of tolerance-related CD4+CD39+Foxp3 T cells (Niedbala et al. 2007; Kochetkova et al. 2010) and disrupted Th17 cell function, thereby alleviating Th1/Th17related inflammatory symptoms (Niedbala et al. 2007; Wirtz et al. 2011).

As with other regulatory immune molecules, IL-35 probably activates the JAK-STAT pathway after binding its cognate receptors (Gee et al. 2009). IL-35 was shown to signal through STAT1 or STAT4 after binding to gp130 or IL12R $\beta 2$ on T cells (Collison et al. 2012). Liver samples from patients with $\mathrm{PBC}$ showed strong staining for IL12R $\beta 2$ and IFN- $\gamma$ in the early stage and IL-23 and IL-17 in the late stage of disease (Yang et al. 2014a). Exposure to IFN- $\gamma$ upregulated expression of IL12R $\beta 2$, leading to a positive feedback loop in response to antigen stimulus (Webb et al. 2015). Based on its immunological functions, IL-35 has been studied in the context of many autoimmune diseases. For instance, patients with RA (Ning et al. 2015) or idiopathic thrombocytopenic purpura (Yang et al. 2014b) showed below-normal serum concentrations of IL-35. In RA, IL-35 was negatively related to the concentration of rheumatoid factor and percentage of neutrophils (Ning et al. 2015). In idiopathic thrombocytopenia purpura, IL-35 concentration was positively correlated with the numbers of circulating platelets and Tregs, but negatively correlated with the number of Th1 cells (Yang et al. 2014b).

In conclusion, IL-35, as an immunosuppressive molecule, could be involved in the pathology of $\mathrm{PBC}$, probably by inhibiting expression of Th1/Th17-related cytokines and inducing expression of Treg-related cytokines, such as TGF- $\beta$. This explanation is illustrated by the finding that the plasma IL-35 concentration was related to the histological stage of the disease and treatment status.

\section{Acknowlegments}

This research was supported by grants from the National 973 Foundation (2013CB531606), the National Science Foundation of China $(81671556,81601406,81501397,31500721$, $81501398,81471605,81401358,81302579,81273282$, $81202353,81701608)$, Shanghai Shenkang Grant (SHDC22014014), the Grant of Shanghai Educational Science (D14017), the Grant of the Army Scientific Research (BWS14J023, 15ZD009,15XD007), and the MJD Founding (MJR20150019).

\section{Conflict of Interest}

The authors declare no conflict of interest.

\section{References}

Alunno, A., Manetti, M., Caterbi, S., Ibba-Manneschi, L., Bistoni, O., Bartoloni, E., Valentini, V., Terenzi, R. \& Gerli, R. (2015) Altered immunoregulation in rheumatoid arthritis: the role of regulatory $\mathrm{T}$ cells and proinflammatory $\mathrm{Th} 17$ cells and therapeutic implications. Mediators Inflamm., 2015, 751793. 
Boonstra, K., Beuers, U. \& Ponsioen, C.Y. (2012) Epidemiology of primary sclerosing cholangitis and primary biliary cirrhosis: a systematic review. J. Hepatol., 56, 1181-1188.

Carey, E.J., Ali, A.H. \& Lindor, K.D. (2015) Primary biliary cirrhosis. Lancet, 386, 1565-1575.

Collison, L.W., Chaturvedi, V., Henderson, A.L., Giacomin, P.R., Guy, C., Bankoti, J., Finkelstein, D., Forbes, K., Workman, C.J., Brown, S.A., Rehg, J.E., Jones, M.L., Ni, H.T., Artis, D., Turk, M.J. \& Vignali, D.A. (2010) IL-35-mediated induction of a potent regulatory $\mathrm{T}$ cell population. Nat. Immunol., 11, 1093-1101.

Collison, L.W., Delgoffe, G.M., Guy, C.S., Vignali, K.M., Chaturvedi, V., Fairweather, D., Satoskar, A.R., Garcia, K.C., Hunter, C.A., Drake, C.G., Murray, P.J. \& Vignali, D.A. (2012) The composition and signaling of the IL-35 receptor are unconventional. Nat. Immunol., 13, 290-299.

Collison, L.W., Workman, C.J., Kuo, T.T., Boyd, K., Wang, Y., Vignali, K.M., Cross, R., Sehy, D., Blumberg, R.S. \& Vignali, D.A. (2007) The inhibitory cytokine IL-35 contributes to regulatory T-cell function. Nature, 450, 566-569.

Corpechot, C., Abenavoli, L., Rabahi, N., Chretien, Y., Andreani, T., Johanet, C., Chazouilleres, O. \& Poupon, R. (2008) Biochemical response to ursodeoxycholic acid and long-term prognosis in primary biliary cirrhosis. Hepatology, 48, 871-877.

Dos Passos, G.R., Sato, D.K., Becker, J. \& Fujihara, K. (2016) Th17 Cells Pathways in Multiple Sclerosis and Neuromyelitis Optica Spectrum Disorders: Pathophysiological and Therapeutic Implications. Mediators Inflamm., 2016, 5314541.

Fenoglio, D., Bernuzzi, F., Battaglia, F., Parodi, A., Kalli, F., Negrini, S., De Palma, R., Invernizzi, P. \& Filaci, G. (2012) $\mathrm{Th} 17$ and regulatory $\mathrm{T}$ lymphocytes in primary biliary cirrhosis and systemic sclerosis as models of autoimmune fibrotic diseases. Autoimmun. Rev., 12, 300-304.

Filaci, G., Fenoglio, D. \& Indiveri, F. (2011) CD8(+) T regulatory/ suppressor cells and their relationships with autoreactivity and autoimmunity. Autoimmunity, 44, 51-57.

Gee, K., Guzzo, C., Che Mat, N.F., Ma, W. \& Kumar, A. (2009) The IL-12 family of cytokines in infection, inflammation and autoimmune disorders. Inflamm. Allergy Drug Targets, 8, 40-52.

Griffiths, L., Dyson, J.K. \& Jones, D.E. (2014) The new epidemiology of primary biliary cirrhosis. Semin. Liver Dis., 34, 318-328.

Guan, S.Y., Leng, R.X., Khan, M.I., Qureshi, H., Li, X.P., Ye, D.Q. \& Pan, H.F. (2017) Interleukin-35: a Potential Therapeutic Agent for Autoimmune Diseases. Inflammation, 40, 303-310.

Hirschfield, G.M. \& Gershwin, M.E. (2013) The immunobiology and pathophysiology of primary biliary cirrhosis. Annu. Rev. Pathol., 8, 303-330.

Imam, M.H. \& Lindor, K.D. (2014) The natural history of primary biliary cirrhosis. Semin. Liver Dis., 34, 329-333.

Kleinewietfeld, M. \& Hafler, D.A. (2013) The plasticity of human Treg and Th17 cells and its role in autoimmunity. Semin. Immunol., 25, 305-312.

Kochetkova, I., Golden, S., Holderness, K., Callis, G. \& Pascual, D.W. (2010) IL-35 stimulation of CD39+ regulatory T cells confers protection against collagen II-induced arthritis via the production of IL-10. J. Immunol., 184, 7144-7153.

Kumar, N.P., Moideen, K., George, P.J., Dolla, C., Kumaran, P. \& Babu, S. (2016) Coincident diabetes mellitus modulates Th1-, Th2-, and Th17-cell responses in latent tuberculosis in an IL-10- and TGF- $\beta$-dependent manner. Eur. J. Immunol., 46, 390-399.

Lacerda, M.A., Ludwig, J., Dickson, E.R., Jorgensen, R.A. \& Lindor, K.D. (1995) Antimitochondrial antibody-negative primary biliary cirrhosis. Am. J. Gastroenterol., 90, 247-249.

Lan, R.Y., Salunga, T.L., Tsuneyama, K., Lian, Z.X., Yang, G.X., Hsu, W., Moritoki, Y., Ansari, A.A., Kemper, C., Price, J.,
Atkinson, J.P., Coppel, R.L. \& Gershwin, M.E. (2009) Hepatic IL-17 responses in human and murine primary biliary cirrhosis. J. Autoimmun., 32, 43-51.

Li, X., Mai, J., Virtue, A., Yin, Y., Gong, R., Sha, X., Gutchigian, S., Frisch, A., Hodge, I., Jiang, X., Wang, H. \& Yang, X.F. (2012) IL-35 is a novel responsive anti-inflammatory cytokine: a new system of categorizing anti-inflammatory cytokines. PLoS ONE, 7, e33628.

Liu, B., Zhang, X., Zhang, F.C., Zong, J.B., Zhang, W. \& Zhao, Y. (2013) Aberrant TGF- $\beta 1$ signaling contributes to the development of primary biliary cirrhosis in murine model. World $J$. Gastroenterol., 19, 5828-5836.

Lleo, A., Maroni, L., Glaser, S., Alpini, G. \& Marzioni, M. (2014) Role of cholangiocytes in primary biliary cirrhosis. Semin. Liver Dis., 34, 273-284.

Mannino, M.H., Zhu, Z., Xiao, H., Bai, Q., Wakefield, M.R. \& Fang, Y. (2015) The paradoxical role of IL-10 in immunity and cancer. Cancer Lett., 367, 103-107.

Mattner, F., Magram, J., Ferrante, J., Launois, P., Di Padova, K., Behin, R., Gately, M.K., Louis, J.A. \& Alber, G. (1996) Genetically resistant mice lacking interleukin-12 are susceptible to infection with Leishmania major and mount a polarized Th2 cell response. Eur. J. Immunol., 26, 1553-1559.

Miao, J., Zhang, K., Lv, M., Li, Q., Zheng, Z., Han, Q., Guo, N., Fan, C. \& Zhu, P. (2014) Circulating Th17 and Th1 cells expressing CD161 are associated with disease activity in rheumatoid arthritis. Scand. J. Rheumatol., 43, 194-201.

Moore, K.W., de Waal Malefyt, R., Coffman, R.L. \& O'Garra, A. (2001) Interleukin-10 and the interleukin-10 receptor. Аnnu. Rev. Immunol., 19, 683-765.

Niedbala, W., Wei, X.Q., Cai, B., Hueber, A.J., Leung, B.P., McInnes, I.B. \& Liew, F.Y. (2007) IL-35 is a novel cytokine with therapeutic effects against collagen-induced arthritis through the expansion of regulatory T cells and suppression of Th17 cells. Eur. J. Immunol., 37, 3021-3029.

Nieuwenhuis, E.E., Neurath, M.F., Corazza, N., Iijima, H., Trgovcich, J., Wirtz, S., Glickman, J., Bailey, D., Yoshida, M., Galle, P.R., Kronenberg, M., Birkenbach, M. \& Blumberg, R.S. (2002) Disruption of T helper 2-immune responses in Epstein-Barr virus-induced gene 3-deficient mice. Proc. Natl. Acad. Sci. USA, 99, 16951-16956.

Ning, X., Jian, Z. \& Wang, W. (2015) Low serum levels of interleukin 35 in patients with rheumatoid arthritis. Tohoku J. Exp. Med., 237, 77-82.

Pylayeva-Gupta, Y. (2016) Molecular pathways: interleukin-35 in autoimmunity and cancer. Clin. Cancer Res., 22, 4973-4978.

Qian, C., Jiang, T., Zhang, W., Ren, C., Wang, Q., Qin, Q., Chen, J., Deng, A. \& Zhong, R. (2013) Increased IL-23 and IL-17 expression by peripheral blood cells of patients with primary biliary cirrhosis. Cytokine, 64, 172-180.

Raphael, I., Nalawade, S., Eagar, T.N. \& Forsthuber, T.G. (2015) $\mathrm{T}$ cell subsets and their signature cytokines in autoimmune and inflammatory diseases. Cytokine, 74, 5-17.

Rong, G., Zhou, Y., Xiong, Y., Zhou, L., Geng, H., Jiang, T., Zhu, Y., Lu, H., Zhang, S., Wang, P., Zhang, B. \& Zhong, R. (2009) Imbalance between $\mathrm{T}$ helper type 17 and $\mathrm{T}$ regulatory cells in patients with primary biliary cirrhosis: the serum cytokine profile and peripheral cell population. Clin. Exp. Immunol., 156, 217-225.

Rosenbloom, J., Castro, S.V. \& Jimenez, S.A. (2010) Narrative review: fibrotic diseases: cellular and molecular mechanisms and novel therapies. Ann. Intern. Med., 152, 159-166.

Saeki, R., Ogino, H., Kaneko, S., Unoura, M. \& Kobayashi, K. (1995) Effects of chenodeoxycholic and ursodeoxycholic acids on interferon-gamma production by peripheral blood mononuclear cells from patients with primary biliary cirrhosis. J. Gastroenterol., 30, 739-744.

Sakaguchi, S., Powrie, F. \& Ransohoff, R.M. (2012) Re-establishing immunological self-tolerance in autoimmune disease. 
Nat. Med., 18, 54-58.

Shindo, M., Mullin, G.E., Braun-Elwert, L., Bergasa, N.V., Jones, E.A. \& James, S.P. (1996) Cytokine mRNA expression in the liver of patients with primary biliary cirrhosis (PBC) and chronic hepatitis B (CHB). Clin. Exp. Immunol., 105, 254-259.

Ueno, A., Ghosh, A., Hung, D., Li, J. \& Jijon, H. (2015) Th17 plasticity and its changes associated with inflammatory bowel disease. World J. Gastroenterol., 21, 12283-12295.

Vignali, D.A. \& Kuchroo, V.K. (2012) IL-12 family cytokines: immunological playmakers. Nat. Immunol., 13, 722-728.

Waluga, M., Kukla, M., Zorniak, M., Kochel-Jankowska, A., Kajor, M., Krzeminski, T. \& Kotulski, R. (2015) Visfatin and TGF-Beta1 in primary biliary cirrhosis and two other common liver diseases. Folia Med. Cracov., 55, 59-70.

Wang, D., Zhang, H., Liang, J., Gu, Z., Zhou, Q., Fan, X., Hou, Y. \& Sun, L. (2010) CD4+ CD25+ but not CD4+ Foxp3+ T cells as a regulatory subset in primary biliary cirrhosis. Cell. Mol. Immunol., 7, 485-490.

Wang, L., Sun, Y., Zhang, Z., Jia, Y., Zou, Z., Ding, J., Li, Y., Xu, X., Jin, L., Yang, T., Li, Z., Sun, Y., Zhang, J.Y., Lv, S., Chen, L., et al. (2015a) CXCR5+ CD4+ T follicular helper cells participate in the pathogenesis of primary biliary cirrhosis. Hepatology, 61, 627-638.

Wang, Y.H., Yang, W., Yang, J.B., Jia, Y.J., Tang, W., Gershwin,
M.E., Ridgway, W.M. \& Lian, Z.X. (2015b) Systems biologic analysis of $\mathrm{T}$ regulatory cells genetic pathways in murine primary biliary cirrhosis. J. Autoimmun., 59, 26-37.

Webb, G.J., Siminovitch, K.A. \& Hirschfield, G.M. (2015) The immunogenetics of primary biliary cirrhosis: a comprehensive review. J. Autoimmun., 64, 42-52.

Wirtz, S., Billmeier, U., McHedlidze, T., Blumberg, R.S. \& Neurath, M.F. (2011) Interleukin-35 mediates mucosal immune responses that protect against T-cell-dependent colitis. Gastroenterology, 141, 1875-1886.

Yamaguchi, T., Wing, J.B. \& Sakaguchi, S. (2011) Two modes of immune suppression by Foxp3(+) regulatory $\mathrm{T}$ cells under inflammatory or non-inflammatory conditions. Semin. Immunol., 23, 424-430.

Yang, C.Y., Ma, X., Tsuneyama, K., Huang, S., Takahashi, T., Chalasani, N.P., Bowlus, C.L., Yang, G.X., Leung, P.S., Ansari, A.A., Wu, L., Coppel, R.L. \& Gershwin, M.E. (2014a) IL-12/Th1 and IL-23/Th17 biliary microenvironment in primary biliary cirrhosis: implications for therapy. Hepatology, 59, 1944-1953.

Yang, Y., Xuan, M., Zhang, X., Zhang, D., Fu, R., Zhou, F., Ma, L., Li, H., Xue, F., Zhang, L. \& Yang, R. (2014b) Decreased IL-35 levels in patients with immune thrombocytopenia. Hum. Immunol., 75, 909-913. 